\title{
Use of critical care resources during the first 2 weeks (February 24-March 8, 2020) of the Covid-19 outbreak in Italy
}

\author{
Tommaso Tonetti ${ }^{*}$ (10 , Giacomo Grasselli ${ }^{2,3}$, Alberto Zanella ${ }^{2,3}$, Giacinto Pizzilli ${ }^{1}$, Roberto Fumagalli ${ }^{4}$, \\ Simone Piva ${ }^{5}$, Luca Lorini ${ }^{6}$, Giorgio lotti ${ }^{7}$, Giuseppe Foti ${ }^{8}$, Sergio Colombo ${ }^{9}$, Luigi Vivona ${ }^{2}$, Sandra Rossi ${ }^{10}$, \\ Massimo Girardis ${ }^{11}$, Vanni Agnoletti ${ }^{12}$, Anselmo Campagna ${ }^{13}$, Giovanni Gordini ${ }^{14}$, Paolo Navalesi ${ }^{15}$, \\ Annalisa Boscolo ${ }^{15}$, Alessandro Graziano ${ }^{15}$, Ilaria Valeri ${ }^{15}$, Andrea Vianello ${ }^{16}$, Danilo Cereda ${ }^{17}$, Claudia Filippini ${ }^{18}$, \\ Maurizio Cecconi ${ }^{19,20}$, Franco Locatelli ${ }^{21}$, Michele Bartoletti ${ }^{22}$, Maddalena Giannella ${ }^{22}$, Pierluigi Viale ${ }^{22}$, \\ Massimo Antonellii ${ }^{23}$, Stefano Nava ${ }^{24}$, Antonio Pesenti ${ }^{1,2+}$ and V. Marco Ranieri ${ }^{3 \dagger}$ on behalf of the COVID-19 \\ Northern Italian ICU Network
}

\begin{abstract}
Background: A Covid-19 outbreak developed in Lombardy, Veneto and Emilia-Romagna (Italy) at the end of February 2020. Fear of an imminent saturation of available ICU beds generated the notion that rationing of intensive care resources could have been necessary.

Results: In order to evaluate the impact of Covid-19 on the ICU capacity to manage critically ill patients, we performed a retrospective analysis of the first 2 weeks of the outbreak (February 24-March 8). Data were collected from regional registries and from a case report form sent to participating sites. ICU beds increased from 1545 to 1989 (28.7\%), and patients receiving respiratory support outside the ICU increased from 4 (0.6\%) to 260 (37.0\%). Patients receiving respiratory support outside the ICU were significantly older [65 vs. 77 years], had more cerebrovascular (5.8 vs. $13.1 \%$ ) and renal (5.3 vs. 10.0\%) comorbidities and less obesity (31.4 vs. 15.5\%) than patients admitted to the ICU. $\mathrm{PaO}_{2} / \mathrm{FiO}_{2}$ ratio, respiratory rate and arterial $\mathrm{pH}$ were higher [165 vs. $244 ; 20$ vs. 24 breath/min; 7.40 vs. 7.46] and $\mathrm{PaCO}_{2}$ and base excess were lower [34 vs. $42 \mathrm{mmHg} ; 0.60$ vs. 1.30] in patients receiving respiratory support outside the ICU than in patients admitted to the ICU, respectively.
\end{abstract}

Conclusions: Increase in ICU beds and use of out-of-ICU respiratory support allowed effective management of the first 14 days of the Covid-19 outbreak, avoiding resource rationing.

Keywords: COVID-19, Acute respiratory failure, ICU, Non-invasive ventilation, Rationing

*Correspondence: tommaso.tonetti@unibo.it

${ }^{\dagger}$ Antonio Pesenti and V. Marco Ranieri contributed equally to the work and should be both considered as "senior author"

${ }^{1}$ Alma Mater Studiorum, Dipartimento Di Scienze Mediche E Chirurgiche, Anesthesia and Intensive Care Medicine, Università Di Bologna, Policlinico

Di Sant'Orsola, Via Massarenti, 940138 Bologna, Italy

Full list of author information is available at the end of the article

\section{Introduction}

Data regarding the impact of Covid-19 outbreak on the capacity of the health-care system to accomplish the need for ICU care are limited. The estimated need for intensive care unit (ICU) admission is variable, ranging between 5.0 [1], 7.0 [2] and 26.1\% [3]. Reported ICU mortality ranges between 4 [3], 26 [4] 61 [2], and 67\% [5]. This extreme variability has been attributed to differences 
in terms of beds availability, staff and organization of intensive care units [6].

On Thursday, 20th February 2020, the first cases of positivity for SARS-CoV-2 were recorded in Lombardy region, northern Italy. Since then, the number of patients with Corona Virus Disease-19 (Covid-19) and acute hypoxemic respiratory failure in three regions of northern Italy (Lombardy, Veneto and Emilia-Romagna) dramatically increased, subsequently leading to the call of a national emergency status [7].

A mathematical model of the occupation of intensive care resources in Italy predicted the saturation of the theoretical availability of beds on the national territory by mid-April 2020 [8]. In order to respond to such predicted growing need for ICU resources, on March 1st the Italian government published a notice, ordering to increase the number of ICU beds (https://www.salute.gov.it/porta le/homeMobile.jsp) and approved a law decree that allocated 845 million euros to the public health service to bring the number of ICU beds for invasive mechanical ventilation to the $14 \%$ of the total hospital beds (https:// www.gazzettaufficiale.it/eli/id/2020/03/09/20G00030/ sg).

Since the spread of the SARS-CoV-2 virus is growing and critical care resources of public health systems are dramatically challenged [9], we reasoned that a better understanding of clinical management and ICU requirements for patients with severe Covid-19 at the very beginning of the outbreak may support resources planning and may help to set effective organizational and clinical interventions for the most seriously affected patients. The objective of the study was therefore to (1) describe the process of expansion of the ICU capacity in response to the Covid-19 outbreak during the first 2 weeks of the pandemics; (2) describe settings and modalities of care of acutely ill Covid-19 patients; (3) compare outcomes between critically ill patients with Covid-19 receiving care in or outside the ICU.

\section{Methods}

We retrospectively studied consecutive critically ill patients with confirmed Covid-19 who were referred to the hospitals of the Lombardy, Veneto and EmiliaRomagna regions during the first 2 weeks of the Italian outbreak (February 24-March 8, 2020).

A confirmed case of Covid-19 was defined as a patient with a positive result on high-throughput sequencing or real-time reverse transcriptase-polymerase chain reaction assay of nasal and pharyngeal swab specimens [10].

In total, 30 hospitals (14 in Lombardy, 9 in EmiliaRomagna and 7 in Veneto) participated in the study. Institutional Review Boards reviewed the protocol and authorized data collection.

\section{Data collection}

Data on ICU beds expansion and on total hospital and ICU admissions were gathered from registries of the regional ICUs coordinators of Lombardy (AP), Veneto (PN) and Emilia-Romagna (VMR) [7].

Moreover, a data collection form was circulated among participating ICUs and de-identified data on patients admitted in the ICU and receiving respiratory support outside the ICU were recorded $24 \mathrm{~h}$ after admission. In particular, demographics, comorbidities and basic physiological data were collected.

\section{System-wide changes to ICU and hospital capacity}

In the initial 14 days of the epidemics in Northern Italy, ICU beds and personnel were made available by closing elective surgical admissions and centralizing to a limited number of single non-Covid-19 hub hospitals all neuro- and cardiac-surgical admissions. Moreover, ordinary availability of ICU beds in the three regions was increased from 1545 to 1989 (28.7\%); in particular, ICU capacity increased by $41.4 \%$ (from 725 to 1025 ), $28.1 \%$ (from 370 to 474 ) and $8.9 \%$ (from 450 to 490 ) in Lombardy, Emilia-Romagna and Veneto, respectively. This was achieved by converting operating rooms, coronary units, step-down units and recovery rooms to fully equipped Covid-19 ICUs. Furthermore, the use of outof-ICU respiratory support in the form of CPAP or NIV [11-13] was extended to many different wards, although initial reports suggested caution in the use of non-invasive respiratory support in Covid-19 patients due to the risk of transmission of infection [14].

\section{Clinical care}

All patients included in the study underwent evaluation by a senior intensivist, who decided according to her/ his clinical judgment and to local protocols whether to treat the patient in a ward under supervision of the ICU team or to admit the patient to the ICU. The criteria for ICU admission were: (a) failure of noninvasive respiratory support, defined as persistent hypoxemia, tachypnea and respiratory distress or development of hypercapnia despite the application of CPAP/NIV; (b) expected imminent need for invasive mechanical ventilation; (c) absence of a do-not-intubate order, as discussed collegially by the intensivist and the ward staff physicians caring for the patient.

At all sites out-of-ICU respiratory support was provided by care teams that included at least (i) a senior clinical staff with certified experience in intensive care medicine available around the clock; (ii) nurse support provided with a nurse/patients ratio ranging from 1:4 
to 1:6; (iii) continuous monitoring of electrocardiogram trace, non-invasive blood pressure, oxygen saturation, and respiratory rate.

Conventional oxygen therapy was referred as applied through Venturi or no-rebreathing masks. Helmets were the interface systematically used to deliver CPAP. NIV was equally delivered through mask and helmets. Highflow oxygen therapy was adopted in some units as an alternative to CPAP.

Classification into oxygen therapy and non-invasive respiratory support followed the rule of the highest degree of support; accordingly, a patient receiving oxygen therapy at first and then escalating to non-invasive support was classified as receiving non-invasive support.

\section{Statistical analysis}

Continuous variables were expressed as medians and interquartile ranges (IQR). Categorical variables were summarized as counts and percentages. No imputation was made for missing data. Statistical analyses were descriptive. Comparisons between groups were made using Wilcoxon rank-sum and Pearson's Chi-square. All tests were 2-tailed and were considered significant if $p<0.05$.

Twenty-eight-day mortality of patients admitted in the ICU through the period February 24-March 8, 2020 and of patients receiving respiratory support outside the ICU through the same period was evaluated using the method of Kaplan-Meier. Cumulative incidence of patients extubated and disconnected from mechanical ventilation was calculated and death was considered a competing event. Patients were followed up until April 5th.

All the analyses were performed with the use of SAS software, version 9.4 (SAS Institute Inc., Cary, NC).

\section{Results}

In the period February 24th-March 8th, registries of the coordinating centers of Lombardy, Emilia-Romagna and Veneto showed that a total of 6378 patients were hospitalized for Covid-19 and a total of 805 were admitted and treated in the ICU (12.6\%).

Data collection forms collected from the participating centers provided information on 542 patients treated in the ICU and on 260 patients who received respiratory support outside the ICU (802 patients in total). Notably, the number of patients receiving respiratory support outside the ICU increased from $4(0.6 \%)$ on February 24 to $260(37.0 \%)$ on March 8 (Fig. 1, top), and the proportion of patients admitted to the ICU declined from the $20.3 \%$ of hospitalized Covid-19 patients on February 24 to the $15.2 \%$ of hospitalized Covid-19 patients on March 8 (Fig. 1, bottom).
Compared to patients admitted to the ICU, patients receiving respiratory support outside the ICU were significantly older, had more cerebrovascular and renal comorbidities and fewer of them were obese. The attending intensivists deemed 189 patients $(72.7 \%$ of the 260 patients treated outside the ICU) as non-eligible for further escalation of respiratory support (i.e., for invasive mechanical ventilation). In patients treated outside the ICU, conventional $\mathrm{O}_{2}$ therapy was applied in the $47.3 \%$ of the cases and non-invasive respiratory support (including NIV, CPAP and high-flow $\mathrm{O}_{2}$ therapy) in the $52.7 \%$, while $81.8 \%$ of ICU patients were intubated. $\mathrm{PaO}_{2} / \mathrm{FiO}_{2}$ ratio, respiratory rate and arterial $\mathrm{pH}$ were higher and $\mathrm{PaCO}_{2}$ and base excess were lower in patients receiving respiratory support outside the ICU than in patients admitted to the ICU. (Table 1).

The infectious disease and the pneumology wards were the most more common locations where out-of-ICU respiratory support was delivered $(47.2 \%$ and $30.9 \%$, respectively) (Table 2). Patients receiving conventional $\mathrm{O}_{2}$ therapy outside the ICU had less cerebrovascular comorbidities and obesity and had significantly higher values of $\mathrm{PaO}_{2} / \mathrm{FiO}_{2}$ and arterial $\mathrm{pH}$ than patients receiving noninvasive ventilatory support outside the ICU (including NIV, CPAP and high-flow $\mathrm{O}_{2}$ therapy). Mortality did not differ between patients receiving conventional $\mathrm{O}_{2}$ therapy and non-invasive respiratory support $(58.8 \%$ vs. $52.0 \%$, respectively; Table 3 ).

Analysis of 28-day mortality showed a proportion of deaths of $47.3 \%$ (260 out of 550 ) in patients treated in the ICU and of $52.1 \%$ (135 out of 259), in patients receiving respiratory support outside the ICU $(p=0.0112)$. Nonsurvivors treated in the ICU died within 11 (6-16) days while in non-survivors receiving respiratory support outside the ICU death occurred within 6 (4-11) days. Fortyfour patients in the ICU group (8.0\%) and 10 patients (3.9\%) in the out-of-ICU group were still hospitalized through April 5th (last day of follow-up).

\section{Discussion}

The present study describes how the Italian health-care system of three northern Italian regions responded to the increasing need for clinical resources for critically ill patients during the first 14 days of the Covid-19 outbreak through the $28.7 \%$ increase in ICU beds and the increasing use of non-invasive respiratory support outside the ICU.

Data to evaluate the impact of Covid-19 outbreak on the capacity of the health-care system to accomplish the need for ICU resources are limited. Xie and coworkers reported that in Wuhan as of Feb 10, 2020, there were about 1000 patients requiring ventilatory support with 120 new patients every day. However, since only $600 \mathrm{ICU}$ 

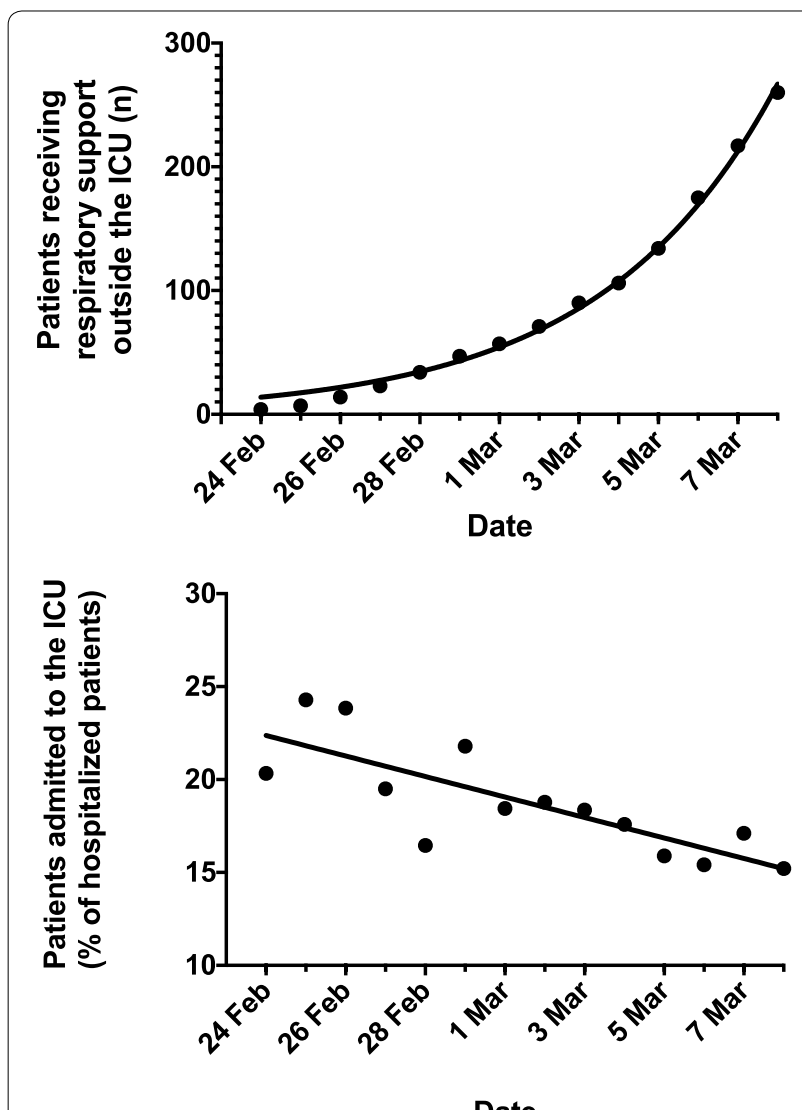

Fig. 1 Number of patients assessed by the intensivist and treated outside the intensive care unit; fitted curve is exponential (top); proportions of patients admitted in the intensive care expressed as percentage of total hospitalized patients; linear fitting (bottom)

beds were available, three general hospitals were rapidly converted to critical care hospitals with a total of about 2500 beds dedicated to Covid-19 critically ill patients [6]. Griffin and coworkers described the process to implement an ICU surge capacity at the greater New York Presbyterian system. In their experience, new COVID-19 ICUs had to be rapidly assembled after the first 3 weeks from the admission of the first critically ill Covid-19 patients [15].

Concomitantly to the increase in ICU bed capacity, there was a progressive increase in the number of patients who received respiratory support outside the ICU (from 0.6 to $37.0 \%$ ) under the daily supervision of an intensivist. This allowed to reduce the percentage of patients admitted to the ICU from 20.3\% on February 24th to $15.2 \%$ on March 8th. The response between the Italian and the greater New York Presbyterian systems was similar, despite the different ICU capacity $(16.2 \%$ of the total hospital beds in the USA [16] vs. $2.8 \%$ in Italy (https://www.salute.gov.it/imgs/C_17_pubblicazi oni_2859_allegato.pdf). This might be explained by the extensive use of out-of-ICU respiratory support we adopted in Italy [11-13].

Our data show that, compared to patients admitted to the ICU, patients receiving respiratory support outside the ICU were significantly older, had more comorbidities and had a higher $\mathrm{PaO}_{2} / \mathrm{FiO}_{2}$ ratio and a lower $\mathrm{PaCO}_{2}$. Among patients treated outside the ICU, proportions of patients treated with conventional $\mathrm{O}_{2}$ therapy and noninvasive respiratory support were comparable (47.3 vs. $52.7 \%$, respectively). The median age of our ICU population [65 years (56-73)] is consistent with the one reported at national level in pre-pandemic times [17] and, although it is difficult to draw conclusion from these data, it is probable that the same age criteria were adopted during the first 2 weeks of the Covid-19 epidemics in Northern Italy.

Patients receiving conventional $\mathrm{O}_{2}$ therapy outside the ICU showed a $\mathrm{PaO}_{2} / \mathrm{FiO}_{2}$ ratio higher than those receiving non-invasive support outside the ICU, without differences in age and mortality. Although a crude comparison of mortality is not very informative because of the baseline differences between the ICU and outof-ICU populations, we show here that the difference in survival at 28 days in patients treated in the ICU and those receiving respiratory support outside the ICU was small ( 47.3 vs. $52.1 \%$, respectively). Altogether these data seem to suggest that treatment outside the ICU has been offered as a therapeutic setting proportional to patient's conditions and not as a 'limited' standard of care, always remaining within the ethical perimeter of standard clinical practice $[18,19]$. Nevertheless, is unlikely that all eligible patients were transferred to an ICU, and we cannot exclude that at least some patients who matched criteria for ICU admission did not survive long enough to be transferred to ICU or comorbid disease or goals of care precluded escalation to ICU level care.

Non-invasive ventilation was suggested to be avoided in Covid-19 patients due to the risk of transmission of infection [14]. In our hospitals, the risk might have been reduced for the following reasons: (a) helmets equipped with high-efficiency particulate air filters at the PEEP port were the interface of choice for delivering non-invasive respiratory support in almost $2 / 3$ of patients treated outside the ICU; this interface might have avoided the dispersion of the multiphase turbulent gas cloud from coughing and sneezing on part of the patients, possibly reducing the transmission of COVID-19[20]; (b) about 50\% of the patients receiving respiratory support outside the ICU were treated in infectious disease wards that are commonly equipped with negative pressure rooms [21]. Moreover, there is growing evidence that NIV can be safely performed outside the ICU in Covid-19 patients, and even advanced maneuvers such as prone positioning have been successfully tested in these patients [22]. 
Table 1 Characteristics of patients receiving respiratory support in the ICU and outside the ICU under intensivist supervision

\begin{tabular}{|c|c|c|c|}
\hline & \multicolumn{2}{|c|}{ Respiratory support } & \multirow[t]{3}{*}{$p$-value } \\
\hline & In the ICU & Outside the ICU & \\
\hline & $N=440$ & $N=260$ & \\
\hline Age (median, IQR) & $65(56-73)$ & $77(65-82)$ & $<0.0001$ \\
\hline Male $(n, \%)$ & $352(80.0)$ & $188(72.3)$ & 0.048 \\
\hline Comorbidities $(n, \%)$ & $N=433$ & $N=260$ & \\
\hline No comorbidities & $73(16.9)$ & $0(0.00)$ & $<0.0001$ \\
\hline COPD & $62(14.3)$ & $39(15.0)$ & NS \\
\hline Diabetes & $103(23.8)$ & $50(19.2)$ & NS \\
\hline Obesity & $136(31.4)$ & $40(15.4)$ & $<0.0001$ \\
\hline HIV infection & $3(0.7)$ & $1(0.4)$ & NS \\
\hline Immunocompromised state & $10(2.3)$ & $9(3.5)$ & NS \\
\hline Cardiovascular disease & $275(63.5)$ & $158(60.8)$ & NS \\
\hline Cerebrovascular disease & $25(5.8)$ & $34(13.1)$ & $<0.001$ \\
\hline Chronic kidney disease & $23(5.3)$ & $26(10.0)$ & 0.0200 \\
\hline Chronic liver failure & $6(1.4)$ & $3(1.2)$ & NS \\
\hline Active neoplasm & $16(3.7)$ & $9(3.5)$ & NS \\
\hline No answer & $100(23.1)$ & $43(16.5)$ & 0.0390 \\
\hline Respiratory support $(n, \%)$ & $N=439$ & $N=241$ & \\
\hline Conventional $\mathrm{O}_{2}$ therapy & $4(0.9)$ & $114(47.3)$ & $<0.0001$ \\
\hline HFNC & $3(0.7)$ & $68(28.2)$ & $<0.0001$ \\
\hline Non-invasive respiratory support & $69(15.7)$ & $59(24.5)$ & 0.0052 \\
\hline CPAP & $28(6.4)$ & $18(7.5)$ & NS \\
\hline NIV & $41(9.3)$ & $41(17.0)$ & 0.0033 \\
\hline Invasive mechanical ventilation & $359(81.8)$ & $0(0.0)$ & $<0.0001$ \\
\hline Physiological variables $^{a}$ & $N=425$ & $N=243$ & \\
\hline $\mathrm{PaO}_{2}$ pressure $(\mathrm{mmHg})$ & $92(76-123)$ & $62(55-73)$ & $<0.0001$ \\
\hline $\mathrm{PaO}_{2} / \mathrm{FiO}_{2}$ ratio & $165(117-230)$ & $244(147-296)$ & $<0.0001$ \\
\hline $\mathrm{PaCO}_{2}(\mathrm{mmHg})$ & $42(37-50)$ & $34(31-38)$ & $<0.0001$ \\
\hline $\mathrm{pH}$ & $7.40(7.34-7.45)$ & $7.46(7.43-7.49)$ & $<0.0001$ \\
\hline Respiratory rate (breath/min) & $20(16-24)$ & $24(18-27)$ & $<0.0001$ \\
\hline Base excess (mEq/L) & $1.30(-1.10-3.90)$ & $0.60(-2.5-2.0)$ & 0.0018 \\
\hline
\end{tabular}

ICU intensive care unit, COPD chronic obstructive pulmonary diseases, HIV human immunodeficiency virus, HFNC high-flow nasal cannula, CPAP continuous positive airway pressure, NIV non-invasive ventilation

${ }^{\text {a }}$ Worst values recorded during the first $24 \mathrm{~h}$ of admission

Table 2 Reported allocations for administering ventilatory support outside the ICU

\begin{tabular}{lc}
\hline Allocation of patients $(\boldsymbol{n}, \boldsymbol{\%})$ & $\boldsymbol{N}=\mathbf{2 5 2}$ \\
\hline Intermediate care unit & $17(6.75)$ \\
Emergency medicine & $38(15.08)$ \\
Infectious disease ward & $119(47.22)$ \\
Pneumology ward & $78(30.95)$ \\
\hline
\end{tabular}

ICU intensive care unit
These data have may important implications for the reorganization required by health-care systems necessary to manage the Covid-19 outbreak. The Italian Society of Anesthesia, Analgesia, Resuscitation, and Intensive Care (SIAARTI) recommended an approach for resource allocation based on "clinical appropriateness" and "distributive justice" in case of significant mismatch between the number of patients requiring ICU admission and the available resources and acknowledged that: "it is not about making choices on value, but to reserve possibly scarce resources first to who has higher probability of survival and second to who can have higher saved years 
Table 3 Clinical and physiological variables of patients receiving respiratory support outside the ICU

\begin{tabular}{|c|c|c|c|}
\hline & $\begin{array}{l}\text { Conventional oxygen therapy } \\
(N=114)\end{array}$ & $\begin{array}{l}\text { Non-invasive respiratory support }{ }^{a} \\
(N=127)\end{array}$ & $p$-value \\
\hline Age & $80(65-83)$ & $75(67-82)$ & NS \\
\hline Male, $n(\%)$ & $72(63.2)$ & $101(79.5)$ & 0.0048 \\
\hline \multicolumn{4}{|l|}{ Comorbidities $(n, \%)$} \\
\hline None & $0(0.0)$ & $0(0.0)$ & \\
\hline COPD & $18(15.8)$ & $21(16.5)$ & NS \\
\hline Diabetes & $19(16.7)$ & $29(22.8)$ & NS \\
\hline Obesity & $23(20.9)$ & $15(11.8)$ & 0.0570 \\
\hline HIV infection & $0(0.0)$ & $0(0.0)$ & \\
\hline Immunocompromised state & $4(3.5)$ & $2(1.6)$ & NS \\
\hline Cardiovascular disease & $73(64.0)$ & $77(60.6)$ & NS \\
\hline Cerebrovascular disease & $20(17.5)$ & $9(7.1)$ & 0.0127 \\
\hline Chronic kidney disease & $13(11.4)$ & $11(8.7)$ & NS \\
\hline Chronic liver failure & $2(1.8)$ & $1(0.8)$ & NS \\
\hline Active neoplasm & $6(5.3)$ & $2(1.6)$ & NS \\
\hline Other & $23(20.2)$ & $17(13.4)$ & NS \\
\hline \multicolumn{4}{|l|}{ Physiological variables ${ }^{b}$} \\
\hline $\mathrm{PaO}_{2}$ pressure $(\mathrm{mmHg})$ & $65(54-77)$ & $59(54-70)$ & 0.0079 \\
\hline $\mathrm{PaO}_{2} / \mathrm{FiO}_{2}$ ratio & $269(198-323)$ & $183(102-265)$ & $<0.0001$ \\
\hline $\mathrm{PaCO}_{2}(\mathrm{mmHg})$ & $35(31-38)$ & $33(30-38)$ & NS \\
\hline $\mathrm{pH}$ & $7.45(7.41-7.48)$ & $7.46(7.44-7.50)$ & 0.0234 \\
\hline Respiratory rate (breath/min) & $24(18-26)$ & $24(20-29)$ & NS \\
\hline Base excess (mEq/L) & $0.50(-2.53 ; 2.15)$ & $0.85(-0.85 ; 2.00)$ & NS \\
\hline 28-day mortality $(n ; \%)$ & $67(58.8)$ & $66(52.0)$ & NS \\
\hline
\end{tabular}

${ }^{a}$ Non-invasive respiratory support includes non-invasive pressure support ventilation, continuous positive airway pressure, high-flow nasal cannula

b Worst values recorded during the first $24 \mathrm{~h}$ of admission

of life, with the purpose of maximizing benefits for the highest possible number of people" [23].

Our data show that increasing the ICU capacity by $28.7 \%$ obtained through the reorganization of available facilities (conversion of operating rooms, coronary units, closure of all scheduled surgical activity) and use of out-of-ICU respiratory support [11-13], the healthcare system was able to accomplish the clinical needs for respiratory support in Covid-19 patients and may suggest that end-of-life practices might have remained within the ethical perimeter of standard clinical practice $[18,19]$.

The retrospective nature represents the major weakness of this study. Although data have been collected by personnel with experience in clinical research and strongly motivated to share their experience, the enormous clinical load and the risk of contagion have certainly influenced the quality of the data and limited the number of information that has been possible to collect. Moreover, further analysis is needed to provide information regarding use of resources, allocation of beds, staffing choices, timing of opening up of new beds, and what resources were most stretched in the first 2 weeks. Moreover, the expected heterogeneity in hospital capacity and care practices between study hospitals may limit the practical utility of the description for clinicians facing an imminent surge of patients with COVID-19 disease. Despite these limitations, this study represents the first and most detailed description of the clinical reality of the first western country overwhelmed by the Covid-19 epidemic.

In conclusion, although our analysis confirms the grave concerns regarding the capacity of health-care systems to effectively respond to the Covid-19 outbreak, these data show that the rapid increase in beds obtained through the reversal of already available resources into intensive care facilities and the use of out-of-ICU respiratory support allowed to manage the first terrible 14 days of the Covid-19 outbreak. The present analysis shows that only rapid acquisition of new intensive care facilities with appropriate equipment and personnel and use of out-ofICU respiratory support [11-13] may avoid the rationing of health-care resources that may be acceptable for "battlefield medicine", but should be incompatible with health-care systems founded on the principles of universality, solidarity and distributive justice (article 32 of the Constitution of the Italian Republic and law number 833 December 23rd, 1978). 


\section{Acknowledgements}

Members of the COVID-19 Northern Italian ICU Network: Lombardy: Giovanni

Albano, Humanitas Gavazzeni, Bergamo (Italy), giovanni.albano@gavazzeni.it; Armando Alborghetti, Policlinico San Pietro-Ponte San Pietro (Italy), armando.alborghetti@grupposandonato.it; Giorgio Aldegheri, IRCCS Multimedica, Sesto San Giovanni (Italy), giorgio.aldegheri@multimedica.it; Benvenuto Antonini, ASST Garda—Ospedale di Manerbio, Manerbio (Italy), benvenuto.antonini@asst-garda.it; Nicolangela Belgiorno, Istituto Clinico San Rocco, Ome (Italy), nicolangela.belgiorno@grupposandonato.it; Giacomo Bellani, ASST Monza - Ospedale San Gerardo, Monza (Italy), giacomo. bellani1@unimib.it; Enrico Beretta, ATS Montagna_Ospedale "Eugenio Morelli" di Sondalo, Sondalo (Italy), enrico.beretta@asst-val.it; Stefano Bonazzi, ASST Bergamo Est—SS Capitanio e Gerosa, Lovere (Italy), stefano.bonazzi@ asst-bergamoest.it; Massimo Borelli, Ospedale Treviglio-Caravaggio, Treviglio (Italy), massimo_borelli@asst-bgovest.it; Luca Cabrini, Ospedale di Circolo e Fondazione Macchi, Varese (Italy), luca.cabrini@uninsubria.it; Livio Carnevale, ASST Pavia_Ospedale di Vigevano, Vigevano (Italy), livio_carnevale@ asst-pavia.it; Sergio Carnevale, IRCCS Ospedale San Raffaele, Milan (Italy), carnevale.sergio@hsrit; Gianpaolo Castelli, ASST Mantova_Ospedale Carlo Poma, Mantova (Italy), gianpaolo.castelli@gmail.com; Emanuele Catena, ASST Fatebenefratelli—Ospedale Sacco, Milan (Italy), emanuele.catena@ asst-fbf-sacco.it; Davide Chiumello, ASST Santi Paolo e Carlo—Ospedale San Paolo, Milan (Italy), chiumello@libero.it; Antonio Coluccello, ASST CremonaOspedale di Cremona, Cremona (Italy), antoniocoluccello@libero.it; Davide Coppini, ASST Garda —Ospedale Civile di "La Memoria", Gavardo (Italy), davide. coppini@asst-garda.it; Daniel Covello, ASST Valle Olona_Ospedale di Busto Arsizio, Busto Arsizio (Italy), daniel.covello@asst-valleolona.it; Gianluca De Filippi, ASST Rhodense_Presidio ospedaliero G. Salvini, Garbagnate Milanese (Italy), gdefilippi@asst-rhodense.it; Marco Dei Poli, IRCCS Policlinico San Donato, Milan (Italy), deipolimd@gmail.com; Paolo Dughi, ASST Franciacorta - Presidio Ospedaliero di Iseo, Iseo (Italy), paolo.dughi@asst-franciacorta.it; Giorgio Gallioli, ASST Vimercate-Ospedale di Vimercate, Vimercate (Italy), giorgio.gallioli@asst-vimercate.it; Paolo Gnesin, ASST Franciacorta_Presidio Ospedaliero Mellino Mellini, Chiari (Italy), paolo.gnesin@asst-franciacorta.it; Stefano Greco, ASST Valle Olona-Ospedale di Saronno, Saronno (Italy), stefano.greco@asst-valleolona.it; Luca Guatteri, Ospedale "Sacra Famiglia" Fatebenefratelli, Erba (Italy), Iuca.guatteri@gmail.com; Davide Guzzon, ASST Lecco_Ospedale di Merate, Merate (Italy), davide.guzzon@asst-lecco.it; Roberto Keim, ASST Bergamo est—Ospedale "Bolognini", Seriate (Italy), roberto.keim@asst-bergamoest.it; Giovanni Landoni, IRCCS Ospedale San Raffaele, Milan (Italy), landoni.giovanni@hsr.it; Thomas Langer, ASST Grande Ospedale Metropolitano Niguarda, Milano, Italy, thomas.langer@unimib.it; Andrea Lombardo, ASST Lariana-Ospedale Sant'Anna, Como (Italy), andrea. lombardo@asst-lariana.it; Giovanni Marino, ASST Melegnano_Ospedale di Vizzolo Predabissi, Melegnano (Italy), giovanni.marino@asst-melegnanomartesana.it; Guido Merli, ASST Crema_Ospedale Maggiore di Crema, Crema (Italy), guido.merli@asst-crema.it; Dario Merlo, ASST Ovest MilaneseOspedale di Magenta, Magenta (Italy), dario.merlo@asst-ovestmi.it; Francesco Mojoli, Fondazione IRCCS Policlinico San Matteo, Pavia, Italy, francesco.mojoli@ unipv.ti; Stefano Muttini, ASST Santi Paolo e Carlo-Ospedale San Carlo, Milan (Italy), stefano.muttini@asst-santipaolocarlo.it; Giuseppe Natalini, Fondazione Poliambulanza Istituto Ospedaliero, Brescia (Italy), giuseppe.natalini@gmail. com; Nicola Petrucci, ASST Garda—Ospedale di Desenzano d/G, Desenzano del Garda (Italy), nicola.petrucci@asst-garda.it; Angelo Pezzi, ASST Nord Milano Ospedale Edoardo Bassini, Cinisello Balsamo (Italy), apezzi67@gmail.com; Danilo Radrizzani, ASST Ovest Milanese—Ospedale Nuovo di Legnano, Legnano (Italy), danilo.radrizzani@asst-ovestmi.it; Maurizio Raimondi, ASST Pavia_Ospedale Civile di Voghera, Voghera (Italy), maurizio_raimondi@ asst-pavia.it; Mario Riccio, Istituti Ospitalieri di Cremona-C.no Ospedale Oglio Po, Casalmaggiore (Italy), m.riccio@asst-cremona.it; Enrico Storti, ASST Lodi-Ospedale Maggiore di Lodi, Lodi (Italy), enrico.storti@asst-lodi.it; Mario Tavola, ASST Lecco—Ospedale di Lecco, Lecco (Italy), ma.tavola@asst-lecco.it; Giovanni Vitale, Policlinico San Marco, Zingonia (Italy), giovanni.vitale@ grupposandonato.it. Veneto: Enrico Bosco, U.O.C. Anestesia e Rianimazione, Ospedale di Conegliano, Conegliano (Italy), enrico.bosco@aulss2.veneto.it; Mauro Antonio Calò, U.O.C. Anestesia e Rianimazione, Presidio Ospedaliero di Mirano, Mirano (Italy), mauroantonio.calo@aulss3.veneto.it; Vinicio Danzi, U.O.C Anestesia e Rianimazione, Ospedale di Vicenza, vinicio.danzi@aulss8.veneto.it; Silvia De Rosa, U.O.C Anestesia e Rianimazione, Ospedale di Vicenza, derosa. silvia@ymail.com; Antonio Farnia, U.O.C. Anestesia e Rianimazione, Ospedale Cà Foncello, Treviso (Italy), antonio.farnia@aulss2.veneto.it; Francesco Lazzari,
U.O.C. Anestesia e Rianimazione, Ospedale dell'Angelo, Venezia Mestre (Italy), francesco.lazzari@aulss3.veneto.it; Marco Meggiolaro, U.O.C. Anestesia e Rianimazione, Ospedale SS. Giovanni e Paolo, Venezia, meggiomarco@libero.it; Marina Munari, U.O.C. Istituto di Anestesia e Rianimazione, Azienda Ospedale Università di Padova, Padova, marina.munari@aopd.veneto.it; Mario Saia, Azienda Zero del Veneto, Padova (Italy), mario.saia@azero.veneto.it; Nicolò Sella, U.O.C. Istituto di Anestesia e Rianimazione, Azienda Ospedale Università Padova, nico.sella@hotmail.it; Eugenio Serra, U.O.C. Istituto di Anestesia e Rianimazione, Azienda Ospedale Università di Padova, Padova, eugenio.serra@ aopd.veneto.it; Ivo Tiberio, U.O.C. Anestesia e Rianimazione, Azienda Ospedale Università Padova, ivo.tiberio@aulss6.veneto.it. Emilia-Romagna: Massimo Baiocchi, Policlinico Sant'Orsola Malpighi, Cardio-Thoracic-Vascular ICU, Bologna (Italy), massimo.baiocchi@aosp.bo.it; Maria Benedetto, Policlinico Sant'Orsola Malpighi, Cardio-Thoracic-Vascular ICU, Bologna (Italy), maria. benedetto@aosp.bo.it; Martina Bordini, Policlinico Sant'Orsola-Malpighi, General ICU, Bologna (Italy), martina.bordini3@studio.unibo.it; Fabio Caramelli, Policlinico Sant'Orsola Malpighi, Bologna (Italy), fabio.caramelli@aosp.bo.it; Nicola Cilloni, Ospedale Maggiore, Bologna (Italy), nicola.cilloni@ausl.bologna. it; Maria Della Giovampaola, Policlinico Sant'Orsola-Malpighi, General ICU, Bologna (Italy), mar.dellagiovampaola@studio.unibo.it; Andrea Fanelli, Policlinico Sant'Orsola Malpighi, Bologna (Italy), andrea.fanelli@aosp.bo.it; Guido Frascaroli, Policlinico Sant'Orsola Malpighi, Cardio-Thoracic-Vascular ICU, Bologna (Italy), guido.frascaroli@aosp.bo.it; Maurizio Fusari, Ospedale "Santa Maria delle Croci", Ravenna (Italy), maurizio.fusari@ausIromagna.it; Giovanni Gordini, Ospedale Maggiore, Bologna (Italy), giovanni.gordini@ausl.bologna.it; Stefano Maitan, Ospedale "G.B. Morgagni L. Pierantoni", Forli (Italy), stefano. maitan@ausIromagna.it; Costanza Martino, Ospedale "M. Bufalini", Cesena (Italy), costanza.martino@auslromagna.it; Raffaele Merola, Policlinico Sant'Orsola-Malpighi, General ICU, Bologna (Italy), raffaele.merola@studio. unibo.it; Rita Maria Melotti, Policlinico Sant'Orsola Malpighi, Università di Bologna, rmelotti@aosp.bo.it; Francesco Minardi, A.O.U. di Parma, Parma (Italy), fminardi@ao.pr.it; Federico Moro, Ospedale Maggiore, Bologna (Italy), mrofrc@ unife.it; Giuseppe Nardi, Ospedale "Infermi", Rimini (Italy), 4doctornardi@gmail. com; Massimo Nolli, AUSL Piacenza, Piacenza (Italy), nolli.massimo@gmail.com; Elisabetta Pierucci, Policlinico Sant'Orsola Malpighi, Bologna (Italy), elisabetta. pierucci@aosp.bo.it; Antonella Potalivo, Ospedale "Infermi", Rimini (Italy), antonella.potalivo@ausIromagna.it; Francesca Repetti, AUSL Piacenza, Piacenza (Italy), francesca.repetti@hotmail.it; Pierpaolo Salsi, Azienda Ospedaliera Santa Maria Nuova, Reggio Emilia (Italy), salsi.pierpaolo@ausl.re.it; Marina Terzitta, Ospedale "G.B. Morgagni L. Pierantoni", Forli (Italy), marina.terzitta@unibo.it; Martina Tosi, A.O.U. di Modena, Modena (Italy), tosimartina@gmail.com; Sergio Venturi, Emergency Commissioner for Emilia-Romagna Region, sergio. venturi@icloud.com; Carlo Alberto Volta, A.O.U. "Arcispedale Sant'Anna”, Ferrara (Italy), vlc@unife.it Gianluca Zani, Ospedale "Santa Maria delle Croci", Ravenna (Italy), gianluca.zani@ausIromagna.it; Andrea Zanoni, Policlinico Sant'Orsola Malpighi, Bologna (Italy), andrea.zanoni@aosp.bo.it.

\section{Authors' contributions}

$T T, G G, A Z, P N, M C, F L, P V, M A, ~ S N$ were responsible for study design, data acquisition, analysis, interpretation, and preparing the first draft of the manuscript. GP, RF, SP, LL, GI, GF, SC, LV, SR, MG, VA, AC, GG, AB, AG, AV, IV, DC, CF, MB $M G$, were responsible for data acquisition and data interpretation. AP and VMR were responsible for study design, data acquisition, analysis, interpretation, finalize the manuscript and study data integrity. All authors had an opportunity to review the manuscript and approved its final submitted version.

\section{Funding}

No funding was provided.

\section{Availability of data and materials}

The datasets used and/or analyzed during the current study are available from the corresponding author on reasonable request.

\section{Ethics approval and consent to participate}

The study was approved by the coordinating center's IRB (Comitato Etico AVEC, Bologna, Italy) with approval number 331/2020/Oss/AOUBo; participant centers obtained approval from their respective IRBs; consent to participate was waived for unresponsive, uncommunicative or deceased patients, in accordance to rule 146/2019 of the Italian Privacy Authority. 


\section{Consent for publication \\ Not applicable.}

\section{Competing interests}

Dr. Cecconi reports personal fees from Edwards Lifesciences, personal fees from Directed Systems, personal fees from Cheetah Medical, outside the present work. Dr. Grasselli reports personal fees and non-financial support from Getinge, personal fees and non-financial support from Biotest, personal fees from Thermofisher, grants and personal fees from Fisher\&Paykel, personal fees from Draeger Medical, outside the present work. Dr. lotti reports personal fees from Hamilton Medical, personal fees from Getinge Italia, personal fees from Eurosets, personal fees from Intersurgical, personal fees from Burke \& Burke, outside the present work. Dr. Pesenti reports personal fees from Maquet, personal fees from Novalung/Xenios, personal fees from Baxter, personal fees from Boehringer Ingelheim, outside the present work. Dr. Zanella has a patent ES2732104 licensed to AW Technologies, a patent US2017348472 licensed to Fresenius, and a patent US2017224898 licensed to Fresenius.

\section{Author details}

${ }^{1}$ Alma Mater Studiorum, Dipartimento Di Scienze Mediche E Chirurgiche, Anesthesia and Intensive Care Medicine, Università Di Bologna, Policlinico Di Sant'Orsola, Via Massarenti, 940138 Bologna, Italy. ${ }^{2}$ Fondazione IRCCS Ca' Granda Ospedale Maggiore Policlinico, University of Milan, Milan, Italy. ${ }^{3}$ Department of Pathophysiology and Transplantation, University of Milan, Milan, Italy. ${ }^{4}$ Anesthesia and Critical Care, ASST Grande Ospedale Metropolitano Niguarda, University of Milano-Bicocca, Milan, Italy. ${ }^{5}$ Anesthesia and Critical Care, ASST Spedali Civili, University of Brescia, Brescia, Italy.

${ }^{6}$ Anesthesia and Critical Care, ASST Papa Giovanni XXIII, Bergamo, Italy. ${ }^{7}$ Anesthesia and Critical Care, Fondazione IRCCS Policlinico San Matteo, Pavia, Italy. ${ }^{8}$ Anesthesia and Critical Care, ASST Ospedale San Gerardo Di Monza, University of Milano-Bicocca, Milan, Italy. ${ }^{9}$ Anesthesia and Critical Care, IRCCS San Raffaele Scientific Institute, Università Vita-Salute San Raffaele, Milan, Italy. ${ }^{10}$ Anesthesia and Critical Care, Azienda Ospedaliero-Universitaria Di Parma, Parma, Italy. ${ }^{11}$ Anesthesia and Critical Care, Policlinico Di Modena, Università Di Modena E Reggio Emilia, Modena, Italy. ${ }^{12}$ Anesthesia and Critical Care Ospedale "M. Bufalini", Cesena, Italy. ${ }^{13}$ Assessorato Cura Della Persona, Regione Emilia-Romagna, Salute e Walfare, Bologna, Italy. ${ }^{14}$ Anesthesia and Critical Care Ospedale, Ospedale Maggiore, Bologna, Italy. ${ }^{15}$ Anesthesia and Critical Care, Department of Medicine, DIMED - University of Padua, University Hospital of Padua, Padua, Italy. ${ }^{16}$ Respiratory Pathophysiology Division University-City Hospital of Padua, Padua, Italy. ${ }^{17}$ Direzione Generale Welfare, Lombardy Region, Milan, Italy. ${ }^{18}$ Dipartimento Di Scienze Chirurgiche, Università Di Torino, Torino, Italy. ${ }^{19}$ Department of Anesthesia and Intensive Care, Humanitas Clinical and Research Center, Humanitas University, Milan, Italy. ${ }^{20}$ Department of Biomedical Sciences, Pieve Emanuele, Milan, Italy. ${ }^{21}$ Department of Pediatric Hematology and Oncology, Sapienza University of Rome, IRCCS Ospedale Pediatrico Bambino Gesù. President of the "Consiglio Superiore Di Sanità", Rome, Italy. ${ }^{22}$ Alma Mater Studiorum, Dipartimento Di Scienze Mediche E Chirurgiche, Infectious Diseases Unit, Università Di Bologna, Sant'Orsola-Malpighi Hospital, University of Bologna, Bologna, Italy. ${ }^{23}$ Dept. of Intensive Care Emergency Medicine and Anesthesia, Fondazione Policlinico Universitario A. Gemelli IRCCS, Università Cattolica del Sacro Cuore, Rome, Italy. ${ }^{24}$ Department of Clinical, Integrated, and Experimental Medicine (DIMES), Respiratory and Critical Care, Sant'Orsola Malpighi Hospital, Bologna, Italy.

Received: 10 June 2020 Accepted: 23 September 2020

Published online: 12 October 2020

\section{References}

1. Wj G, Ni Zy HuY, Liang Wh, Cq Ou, Jx He, et al. Clinical characteristics of coronavirus disease 2019, in china. N Engl J Med. 2020. https://doi.org/10.1056/ NEJMoa2002032.

2. Yang X, Yu Y, Xu J, Shu H, Xia J, Liu H, et al. Clinical course and outcomes of critically III patients with Sars-Cov-2 pneumonia In Wuhan, China: a singlecentered, retrospective, observational study. Lancet Respir Med. 2020. https ://doi.org/10.1016/S2213-2600(20)30079-5.

3. Wang D, Hu B, Hu C, Zhu F, Liu X, Zhang J, et al. Clinical characteristics of 138 hospitalized patients with, 2019 novel coronavirus-infected pneumonia In Wuhan China. JAMA. 2020. https://doi.org/10.1001/jama.2020.1585.
4. Grasselli G, Zangrillo A, Zanella A, Antonelli M, Cabrini L, Castelli A, et al. Baseline characteristics and outcomes of 1591 patients infected with SarsCov-2 admitted to icus of the Lombardy Region Italy. JAMA. 2020. https:// doi.org/10.1001/jama.2020.5394.

5. Arentz M, Yim E, Klaff L, Lokhandwala S, Riedo FX, Chong M, et al. Characteristics and outcomes of 21 critically III Patients with covid-19 in Washington State. JAMA. 2020. https://doi.org/10.1001/jama.2020.4326.

6. Xie J, Tong Z, Guan X, Du B, Qiu H, Slutsky AS. Critical care crisis and some recommendations during the covid-19 epidemic in China. Intensive Care Med. 2020. https://doi.org/10.1007/s00134-020-05979-7.

7. Grasselli G, Pesenti A, Cecconi M. Critical care utilization for the covid-19 outbreak in Lombardy, Italy: early experience and forecast during an emergency response. JAMA. 2020. https://doi.org/10.1001/jama.2020.4031.

8. Remuzzi A, Remuzzi G. Covid-19 and Italy: what next? Lancet. 2020. https:// doi.org/10.1016/S0140-6736(20)30627-9.

9. Rosenbaum L. Facing covid-19 in Italy-ethics, logistics, and therapeutics on the epidemic's front line. N Engl J Med. 2020. https://doi.org/10.1056/ NEJMp2005492.

10. Huang C, Wang Y, Li X, Ren L, Zhao J, Hu Y, et al. Clinical features of patients infected with 2019 novel coronavirus in Wuhan China. Lancet. 2020;395(10223):497-506.

11. Squadrone V, Massaia M, Bruno B, Marmont F, Falda M, Bagna C, et al. Early Cpap prevents evolution of acute lung injury in patients with hematologic malignancy. Intensive Care Med. 2010;36(10):1666-744.

12. Priestley G, Watson W, Rashidian A, Mozley C, Russell D, Wilson J, et al. Introducing critical care outreach: a ward-randomised trial of phased introduction in a general hospital. Intensive Care Med. 2004;30(7):1398-404.

13. Ball C, Kirkby M, Williams S. Effect of the critical care outreach team on patient survival to discharge from hospital and readmission to critical care: non-randomised population based study. BMJ. 2003;327(7422):1014.

14. Van Doremalen N, Bushmaker T, Morris DH, Holbrook MG, Gamble A, Williamson BN, et al. Aerosol and surface stability of Sars-Cov-2 as compared with Sars-Cov-1. N Engl J Med. 2020;382(16):1564-7.

15. Griffin KM, Karas MG, Ivascu NS, Lief L. Hospital preparedness for covid-19: a practical guide from a critical care perspective. Am J Respir Crit Care Med. 2020;201(11):1337-444.

16. Wallace DJ, Angus DC, Seymour CW, Barnato AE, Kahn JM. Critical care bed growth in the United States: a comparison of regional and national trends. Am J Respir Crit Care Med. 2015;191(4):410-6.

17. Sakr Y, Elia C, Mascia L, Barberis B, Cardellino S, Livigni S, et al. The influence of gender on the epidemiology of and outcome from severe sepsis. Crit Care. 2013;17(2):R50.

18. Sprung CL, Ricou B, Hartog CS, Maia P, Mentzelopoulos SD, Weiss M, et al. Changes in end-of-life practices in european intensive care units from 1999 to 2016. JAMA. 2019;89:1-12.

19. Guidet B, Flaatten H, Boumendil A, Morandi A, Andersen Fh, Artigas A, et al. Withholding or withdrawing of life-sustaining therapy in older adults $(>/=80$ years $)$ admitted to the intensive care unit. Intensive Care Med. 2018;44(7):1027-38

20. Bourouiba L. Turbulent gas clouds and respiratory pathogen emissions: potential implications for reducing transmission of covid-19. JAMA. 2020. https://doi.org/10.1001/jama.2020.4756.

21. Ferioli M, Cisternino C, Leo V, Pisani L, Palange P, Nava S. Protecting healthcare workers from Sars-Cov-2 infection: practical indications. Eur Respir Rev. 2020:29:155.

22. Sartini C, Tresoldi M, Scarpellini P, Tettamanti A, Carco F, Landoni G, et al. Respiratory parameters in patients with covid-19 after using noninvasive ventilation in the prone position outside the intensive care unit. JAMA. 2020;8:123.

23. http://www.Siaarti.lt/Siteassets/News/Covid19\%2520-\%2520docume nti\%2520siaarti/Siaarti\%2520-\%2520covid-19\%2520-\%2520clinical\%2520e thics\%2520reccomendations.Pdf.

\section{Publisher's Note}

Springer Nature remains neutral with regard to jurisdictional claims in published maps and institutional affiliations. 